\title{
Estimating the effects of access time windows in the management of urban delivery fleets
}

\author{
R. Grosso, J. Muñuzuri, M. Rodríguez Palero \& P. Aparicio \\ University of Seville, Spain
}

\begin{abstract}
The analyses prior to the introduction of access time window policies in the centre of European cities often neglect the evaluation of the extra costs imposed on carriers through the additional number of vehicles required and the increase in tour length. To facilitate this evaluation, we have developed a vehicle routing algorithm that takes into account the existence of access time windows and adapts tours in the best possible manner to this restriction. The algorithm is based on a Genetic Algorithm, which we use to make this evaluation through the analysis of several experiments in a test network.
\end{abstract}

Keywords: vehicle routing, access time windows, city logistics, Genetic Algorithms.

\section{Introduction}

Vehicle routing problems constitute one of the most widespread topics in scientific literature. Academic papers, occasionally resulting in commercial software applications, provide a long list of problem setups and solution techniques, from linear optimization to the most advanced and modern metaheuristic approaches. However, the list of vehicle routing problem types continues to increase, mainly due to the ever-increasing complexity of fleet management scenarios. In fact, the main concern of this paper lies with the definition of one of these complex transportation scenarios, often found in urban freight deliveries, rather than with the methodological search of the best possible solution method.

The problem formulated here stems, like all the other routing problems, from the observation of reality, and more specifically from the difficulties encountered by freight delivery companies operating in urban areas. Sustainability policies 
lead local authorities to increasingly restrict the access of vehicles to the central area of cities by means of pedestrianisation schemes, parking space elimination or access restrictions (Anderson et al. [1]). These access restrictions are often applied in the form of time restrictions, establishing time windows for passenger cars and delivery vehicles to access the restricted area. These mobility restrictions, especially in Europe, have a significant effect on freight operators, given that the density of commercial premises, and therefore of freight deliveries, is much higher in central areas, and besides these time windows are normally closed during most part of the daily business hours, the only time when retail freight receivers are accepting deliveries.

This type of time window differs from the ones found in the VRPTW in that it is not imposed by the customer, but the local authorities. Besides, the time window is not only related to accessing the customer's premises, but in general to the overall restricted area, also forbidding delivery vehicles to cross it or wait inside of it, even if no delivery operations are in progress. In short, there is a relatively large area of the city subject to access restrictions under a predefined timetable, which has to be taken into account by transport operators when planning their daily routes. We will refer to this planning process as a Vehicle Routing Problem with Access Time Windows (VRPATW), which combines the zonal and the time factors affecting equally all the customers established inside the restricted area.

Once again, the introduction of these access time window restrictions by local authorities responds to sustainability criteria, seeking to eliminate congestion and pollution from the central areas of cities during the most sensible time of the day. However, the analyses related to the introduction of these policies have failed to take into account an amount of relevant inputs (Seasons [2]). For instance, the extra costs imposed on freight transport companies, forcing them to use an increasing number of extra vehicles depending on the size of the restricted area and on the duration of the time window. We have taken this logistics and economic point of view in our analysis, and sought to estimate those extra costs with a double objective: to communicate them to local authorities so that they can be taken into account in the corresponding cost-benefit analyses (Vickerman [3]) when introducing access time window policies, and to help transport companies deal with those policies in their daily operation.

In terms of the solution approach, we have used a standard Genetic Algorithm to determine the effect of access time windows on fleet routing and scheduling. Our objective here was not methodological but practical, using this algorithm simply as a tool to estimate the effects of the time window policy and to be able to draw some conclusions with respect to it. Algorithmically, we believe the main methodological contribution lies in the computation of the fitness function, specifically designed for the characteristics of the VRPATW.

\section{Problem description}

We consider our vehicle routing problem defined on a graph: $[N, L]$, where $N$ is the set of nodes and $L$ is the set of links communicating them. The set of nodes $N$ 
contains one node $d$ with a positive level of supply (depot), a subset $C$ of nodes with a positive level of demand (customers), and another subset $\bar{C}$ of nodes with zero levels of supply and demand, so that $N=(C \cup \bar{C}) \cup d$. A number $V$ of vehicles (where $V$ is a variable) will travel through the graph visiting all the different customers, only one vehicle per customer. We do not consider capacity restrictions on vehicles, which is a realistic assumption in the case of less-thantruckload urban freight deliveries, where vehicles are rarely full.

The problem is defined inside a predefined time horizon, corresponding to the day's working hours, and the objective is to minimize the number of vehicles that need to be used and the cost (in time units) of transporting goods from the depot $\mathrm{d}$ to the nodes of $C$, crossing along the way the necessary nodes of the subset $\bar{C}$.

We also define a set $T$ of time costs associated to the different links in the graph. These costs depend only on the transit of vehicles through links, and not on the amount of freight carried by those vehicles. In general, we will incur in cost $t_{i j}$ when travelling from node $i$ to node $j$. We will also compute the unloading time at each customer as a time cost $h$, incurred every time a vehicle visits one of the customer nodes contained in $C$.

Within the set of nodes $N$, we also consider a subset $R Z$ of nodes that correspond to the restricted zone, and which cannot be crossed or visited during a pre-specified closed time window period (CWT), which period will obviously be smaller than the overall time horizon. We assume that $C \cap R Z \neq \varnothing$ and that $\bar{C} \cap R Z \neq \emptyset$.

A schematic description of the problem is shown in Figure 1.

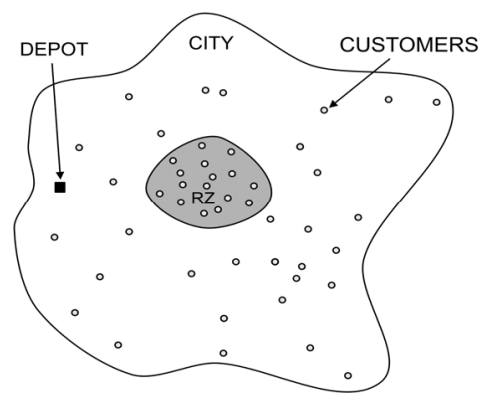

Figure 1: $\quad$ Schematic representation of a city where a VRPATW applies.

Before describing the different situations that have to be considered in the routing process, we define here the concepts and terminology involved. For example, with respect to the time window:

- $t_{V C} \equiv$ Window closing time, that is, the instant when the time window restriction starts operating.

- $t_{V A} \equiv$ Window opening time, that is, the instant when the time window restriction ends. 
With respect to the time horizon, linked to a full day or rather the business hours of a given day, we define:

- $t_{o} \equiv$ Starting time for the day.

- $t_{f} \equiv$ End-of-day time, when the business hours end.

All these instants are represented in the timeline shown in Figure 2.

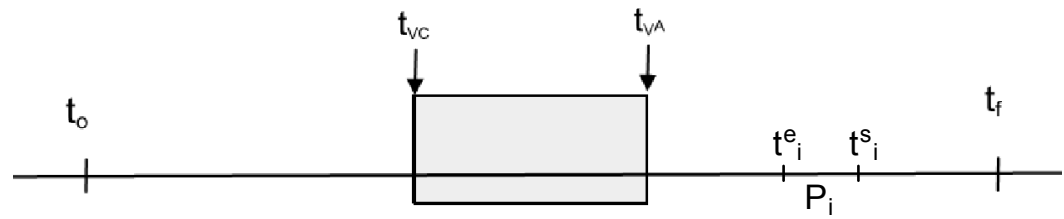

Figure 2: Timeline showing the start and end of the time horizon, and the start and end of the time window access restriction. Representation of the times of arrival and departure at customer $i$ in a vehicle's route.

The cost models the fact that the vehicle, when visiting a customer, needs to spend a given amount of time unloading the goods and making the final delivery. This is why we distinguish a time of arrival and a time of departure for all the nodes in subset $\mathrm{C}$ for the analysis and interpretation of the problem. The terminology used is as follows:

- $\quad t_{i}^{e} \equiv$ Arrival time to customer $i$ for the vehicle visiting that customer.

- $\quad t_{i}^{S} \equiv$ Departure time from customer $i$ towards customer $i+1$, with $t_{i}^{S}=$ $t_{i}^{e}+h$.

Going back to the timeline in Figure 2, we have represented these two times, with $P_{i}$ representing customer or stop $i$ in the route of a vehicle.

\section{Case analysis}

After defining the problem, the time window, the restricted zone and the related terminology, we now proceed to analyze the different cases that may arise when calculating vehicle routes. Starting with the general scenario of a vehicle moving from one customer to the next one until the end of its route, we will describe those cases, their interaction with the time window and the restricted zone, and what should be the reaction of the algorithm in each one of them.

\subsection{General case}

If a vehicle has to visit a determined sequence of customers, the default situation establishes that it starts its route at the depot at the starting time of the day $t_{o}$. Then, at every customer that is visited, we calculate the corresponding arrival and departure time, but we also need to take into account whether the customer in inside the restricted zone and, if so, whether the time window is open or closed. If no restrictions affect the displacement, the process continues with the following customer in the sequence, until all the customers have been visited and 
the end of the day is reached for the vehicle. The process is the same for all the different vehicles introduced in the routing planning.

However, if any restriction related to the time window could affect the visit to a given customer in the route, additional considerations must be introduced. These considerations, and the procedure followed in each case, are described in the following sections.

\subsection{Entering the restricted zone}

This is the case of traveling from customer $i$, located outside of the restricted zone $\mathrm{RZ}$, to customer $i+1$, located inside. Several different situations can appear in this case, depending on whether the time window is open or closed. If the time window is open at the time of arrival at customer $i+1, t_{i+1}^{e}$, the procedure is identical to the general case, since the time window does not affect the displacement.

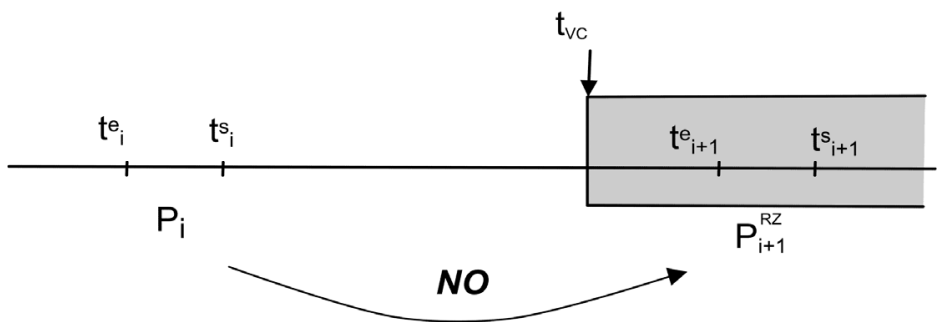

Figure 3: Representation of the entry to the restricted zone when the time window is closed.

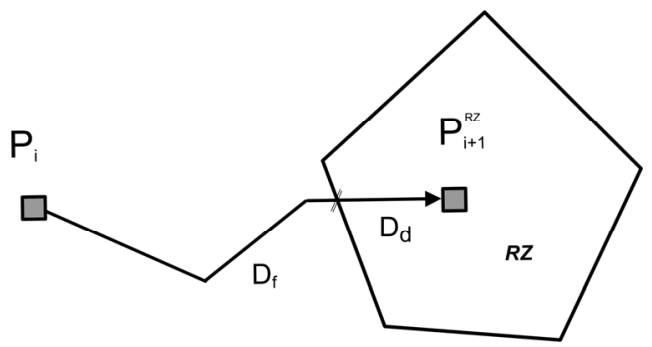

Figure 4: Distances covered inside and outside the restricted zone when travelling between customers $i$ and $i+1$.

However, if the time window is closed at $t_{i+1}^{e}$, the displacement is unfeasible (see Figure 3), and the distance (in time units) covered between both customers needs to be divided (see Figure 4) into $D_{f}$ (distance covered outside of the restricted zone) and $D_{d}$ (distance covered inside the restricted zone).

In this case, the vehicle needs to wait at the previous customer until the time window opens again and it can access the restricted zone. If that previous customer is not located inside the restricted zone, the instant of arriving at 
customer $i+1$ is moved forward, making $t_{i+1}^{e}$ equal to $t_{V A}+D_{d}$ (see Figure 5). This synchronizes the instant of entering the restricted zone with the instant when the time window is open again, thus guaranteeing that the vehicle is never inside the restricted zone while the time window is closed.

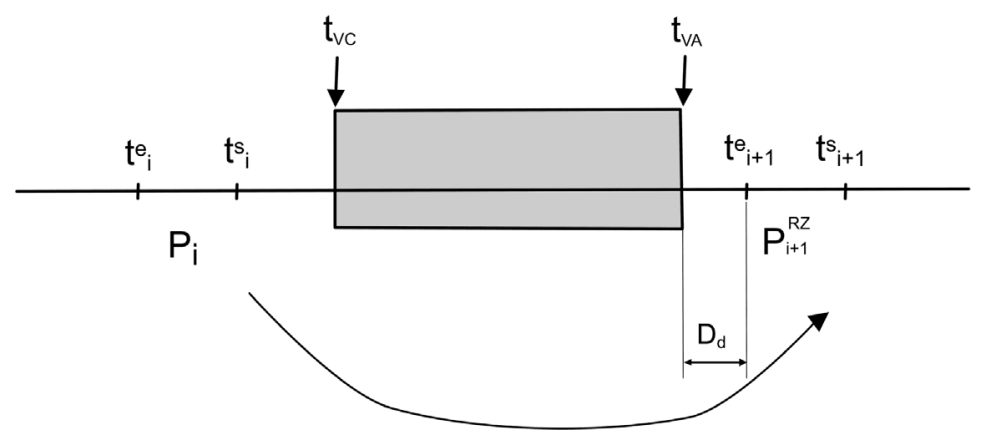

Figure 5: Readjustments of the arrival time to customer $i+1$ in order to ensure that the vehicle is not inside the restricted zone while the time window is closed.

Next, and seeking to reduce the overall route duration, the arrival time to the previous customer $i$ also needs to be revised, moving it forward as close as possible to the new arrival time to customer $i+1$. This mechanism is represented in Figure 6.

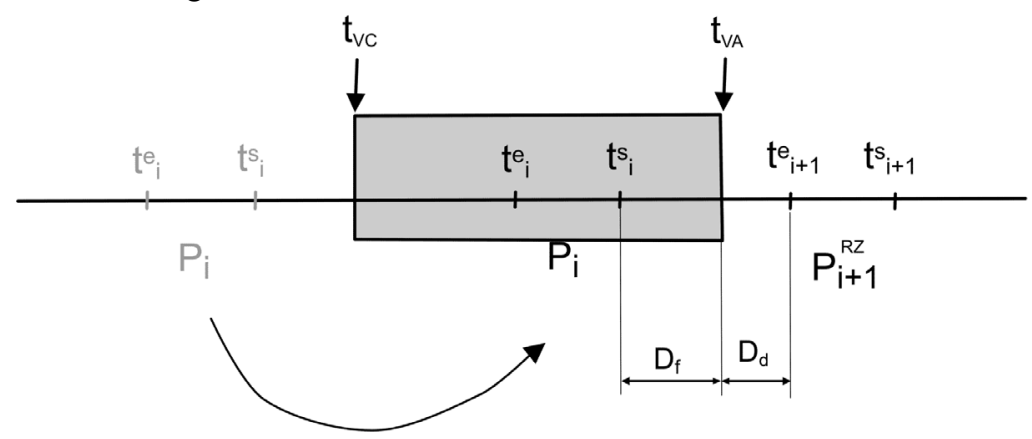

Figure 6: $\quad$ Readjustment of the arrival time to customer $i$ as close as possible to the new arrival time to customer $i+1$.

This readjustment can in turn result in different scenarios in case the customers visited before customer $i+1$ are also located inside the restricted zone. In that case, the route planning procedure moves this customer/s located inside the restricted zone to visit them after the time window opens and waits in a customer located outside the restricted zone until the time window is open again always seeking to reduce the overall route duration. 


\subsection{Leaving the restricted zone}

If the vehicle is now travelling from customer $i$, located inside the restricted zone, to customer $i+1$, located outside, we can again distinguish between two parts of the trajectory, one inside the restricted zone $\left(D_{d}\right)$ and the other one outside $\left(\mathrm{D}_{\mathrm{f}}\right)$, as shown in Figure 7.

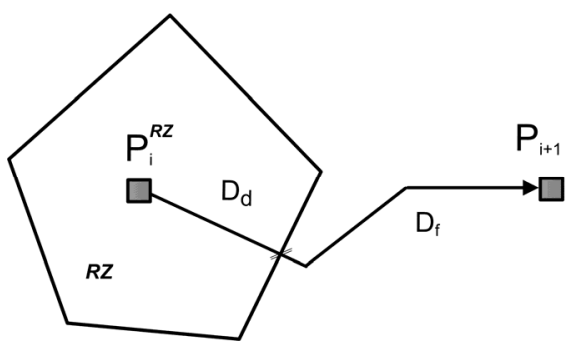

Figure 7: Illustration of the displacement between a customer located inside the restricted zone to another one located outside.

The possible cases to contemplate now, depending on the time window, are very similar to the previous scenario. For instance, if the time window is open when reaching customer $i+1$, the displacement follows the general case procedure, as shown in Figure 8.

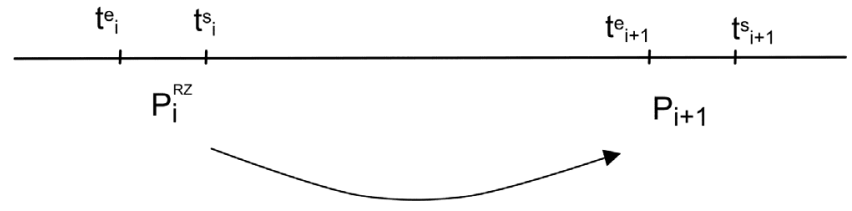

Figure 8: $\quad$ Exiting the restricted zone while the time window is open.

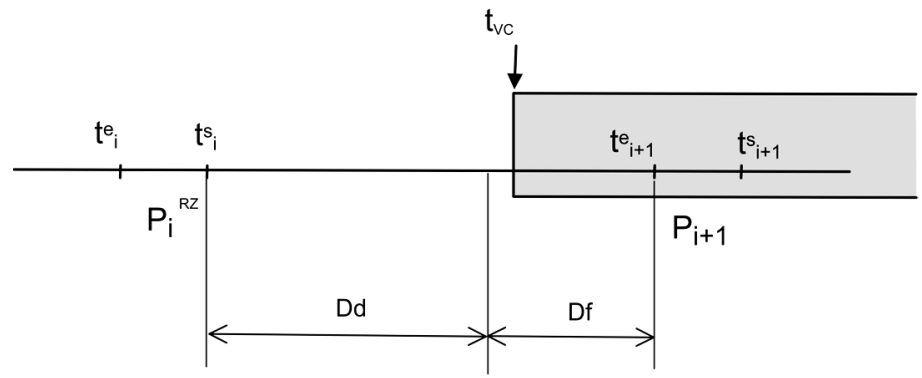

Figure 9: Feasible displacement, since the vehicle leaves the restricted zone before the time window closes.

However, the time window may close on the vehicle during the displacement, which happens when the vehicle leaves the restricted zone after $t_{V A}$. For instance, the displacement depicted in Figure 9 is valid, and is treated like the general case, whereas the one in Figure 10 is not. 


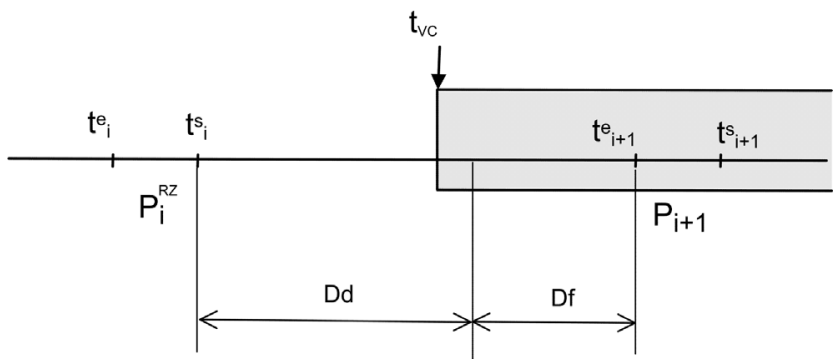

Figure 10: Unfeasible displacement, since the vehicle would leave the restricted zone after the time window closes.

In this unfeasible case, the procedure needs to readjust the arrival time to customer $i$, moving it back until the time window is open again (see Figure 11). Then, like in the previous scenarios, all the customers visited before $i$ and which are not located inside the restricted zone would also be moved back in order to visit them while the time window is closed and thus save time in the overall route.

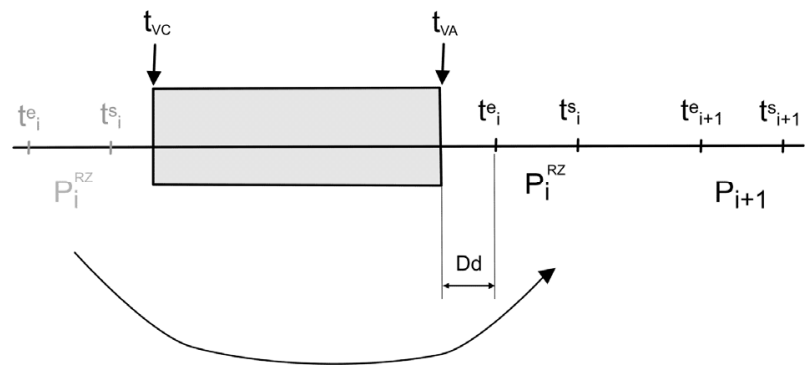

Figure 11: Readjustment of the arrival time to customer $i$ as close as possible to the re-opening of the time window.

\section{The genetic algorithm}

The algorithm used to solve the VRPATW is based on the sequence of stops provided by a basic Genetic Algorithm, built according to the design shown in Figure 12. The operators of this GA can be briefly described as follows:

- Crossover: built according to the evaluative procedure proposed by Uchimura and Sakaguchi [4].

- Mutation: random selection and exchange of two stops in the sequence.

- Probabilistic selection: assigning a probability of survival linearly distributed between 0 and 1 depending on the fitness of the individual (which depends on the total number of vehicles used and the total duration of the routes). 
- Population restart: when the best fitness value is less than 5\% below the average fitness value, keeping only the three best individuals of the population.

- The stopping criterion is only associated to the number of iterations.

- Fitness: specifically designed for the characteristics of the VRPATW is based on the discussion in section 3 .

The flowchart of this procedure is shown in Figure 12.

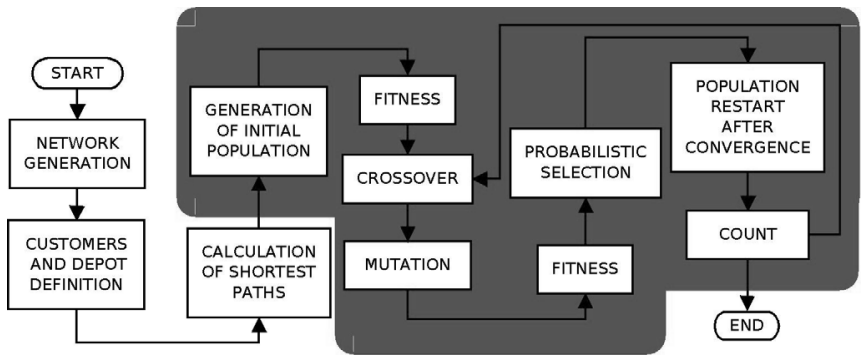

Figure 12: General structure of the genetic algorithm used.

\section{Test problem}

\subsection{Description of the test network}

For the implementation of the algorithm, we used a test network (see Figure 13) with a simple design that enabled us to interpret the results and analyze them. The test network consisted of 900 nodes distributed in a 30x30 square and communicated by links of equal length. Figure 13 shows the direction distribution for the links in the test network, alternating in the different rows and columns of the squared pattern. In the experiments were built taking into account that the depot must be placed outside the restricted zone, and that nodes inside the restricted zone have a higher probability of containing a customer, which corresponds to reality.

When solving the test problems, the evaluation of the population's fitness applied the following hypotheses and restrictions:

- The maximum number $V$ of vehicles in the fleet is infinite; we assumed that it was in any case sufficient to cover all the necessary deliveries.

- We established a fixed cost $n v=8000$ time units for each additional vehicle, apart from the first one, that is required to complete the deliveries inside the time horizon. This penalizes the introduction of new vehicles in the solution.

- The length of the links in the test network is assumed to be equal to $200 \mathrm{~m}$.

- We assigned a constant speed equal to $20 \mathrm{Km} / \mathrm{h}$ for delivery vehicles. This resulted in the determination of the time costs $t_{i j}$ corresponding to each link. In the case of the test network, all these costs are equal. 
- The time horizon is equal to $\left[t_{o}, t_{f}\right]=10$ hours.

- The time window is supposed to be centered in the time horizon, located in the middle of the day.

- The unload time, or the time that delivery vehicles spend at each customer, is $h=20$ minutes.

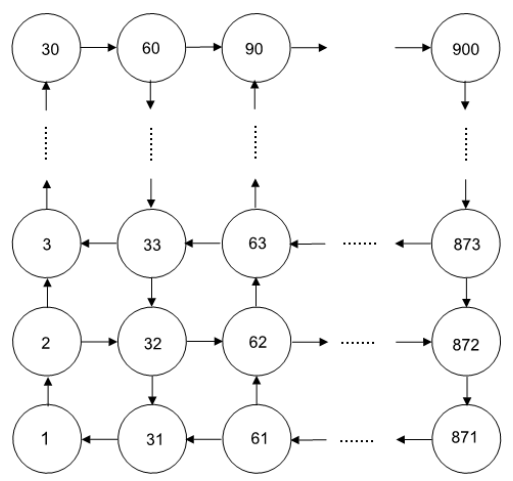

Figure 13: Schematic representation of the test network used to calibrate the algorithm.

\subsection{Experiments and results}

The experiments were built according to the values of three parameters related to the problem and three more related to the GA, as follows:

- Parameters of the problem:

- Number of customers ( $\mathrm{N}^{\mathrm{o}}$ Stop)

$\circ \quad$ Size of the restricted zone (RZ), measured in length units, with each unit equivalent to 50 meters.

- Length of the time window (TW), measured as the number of hours during which the time window is closed.

- Parameters of the Genetic Algorithm:

- Population size (A): the population size is a function of the number of customers in the problem, multiplied by this parameter: Population size $=A \times N^{\circ}$ Stop.

○ Maximum number of iterations (B): the number of iterations for the GA also depends on the number of customers: $N^{o}$ Iterations $=B \times N^{o}$ Stop.

- Probability of mutation.

This resulted in 9 different problems with different values of the number of customers $(40,120)$, the size of the restricted zone (considering no restricted zone or $\mathrm{ZN}$ equal to 20 - the current scenario - 50) and the length of the time windows restriction (4 and 8). For each number of customers, their location is always the same, varying only the size of the restricted zone and the length of the time window. This problem was solved with 8 different configurations of the GA. Table 1 shows the values of the parameters in each one of the 72 
experiments thus built, together with the fitness value for the best solution found in each case and the number of delivery vehicles used. Written in bold are the lowest fitness values for each type of problem.

Table 1: $\quad$ Results of the experiments solved on the test problem.

\begin{tabular}{|c|c|c|c|c|c|c|c|c|c|c|c|c|c|c|c|c|c|c|c|}
\hline A & 吕 $\frac{\stackrel{0}{0}}{\text { क }}$ & $\stackrel{N}{\alpha}$ & 3 & \multicolumn{2}{|c|}{$\begin{array}{c}\text { Exp. } 1 \\
0,5\end{array}$} & \multicolumn{2}{|c|}{$\begin{array}{c}\text { Exp. } 2 \\
0,5\end{array}$} & \multicolumn{2}{|c|}{$\begin{array}{c}\text { Exp.3 } \\
0,5\end{array}$} & \multicolumn{2}{|c|}{$\begin{array}{c}\text { Exp.4 } \\
0,5\end{array}$} & \multicolumn{2}{|c|}{$\begin{array}{c}\text { Exp. } 5 \\
2 \\
\end{array}$} & \multicolumn{2}{|c|}{$\begin{array}{c}\text { Exp.6 } \\
2\end{array}$} & \multicolumn{2}{|c|}{$\begin{array}{c}\text { Exp.7 } \\
2 \\
\end{array}$} & \multicolumn{2}{|c|}{$\begin{array}{c}\text { Exp.8 } \\
2\end{array}$} \\
\hline $\begin{array}{c}\text { B } \\
\text { TM }\end{array}$ & & & & 3 & & 0,5 & & 0,1 & & 0,5 & & 0,1 & & $\begin{array}{c}3 \\
0,5\end{array}$ & & $\begin{array}{c}6 \\
0,1\end{array}$ & & $\begin{array}{c}6 \\
0,5\end{array}$ & \\
\hline $\mathrm{P} 1$ & 50 & & & 14288 & & 4304 & & 14232 & & 14256 & & 14264 & & 14280 & & 4208 & & 4224 & 2 \\
\hline P2 & 50 & 20 & 4 & 38 & 3 & 22922 & & 15680 & & 22456 & & 14296 & 2 & 2688 & 3 & 14312 & 2 & 4112 & \\
\hline P3 & 50 & 20 & 8 & & & 000 & & 45608 & & 50568 & & 4850 & & 834 & & 44858 & & 170 & \\
\hline $\mathrm{P}$ & 0 & 50 & 4 & 580 & 3 & 23614 & 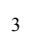 & 23448 & 3 & 23690 & 3 & 23508 & 3 & 23470 & 3 & 23326 & 3 & 5218 & 2 \\
\hline P5 & 50 & 50 & 0 & 44 & & 80526 & & 80424 & & 80452 & & 702 & 7 & 740 & & 0062 & & 76982 & 8 \\
\hline $\mathrm{P} 6$ & & 20 & 4 & 4 & & 896 & 6 & 696 & 6 & 854 & 5 & 832 & 5 & 110 & 5 & 634 & 5 & 6656 & 5 \\
\hline P7 & 120 & 20 & 8 & & & 3302 & & 4258 & & 9314 & & 126514 & & 126514 & & 125238 & & 124962 & 12 \\
\hline P8 & 20 & 50 & 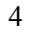 & & & 67776 & & 56642 & 年 & 57152 & & 57636 & 0 & 49878 & 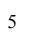 & 6 & 0 & 184 & 6 \\
\hline P9 & 120 & 50 & 8 & 07640 & 91 & 198746 & 91 & 195420 & 19 & 186762 & 8 & 179020 & 16 & 193020 & 18 & 170308 & 16 & 175548 & 17 \\
\hline
\end{tabular}

\section{Conclusions}

In the previous section, we have not provided computation times for the solution of the different problems. The solution of these VRPATW is very timeconsuming due to the complicated computation of the fitness functions, but our main objective was not the optimization of the daily planning of these routes, but rather the estimation of the extra costs imposed on carriers with the implementation of access time window policies through the analysis of a test problem. This test problem provides several interesting findings:

- The scenarios with the current size of the restricted zone $(R Z=20)$ show small cost increments with respect to the base (no-window) scenario, but these increments grow larger as the length of the time window (TW) increases.

- The scenarios with $\mathrm{RZ}=50$ do show significant differences with respect to the base scenario like a large increase in the number of vehicles required in the fleet, which represents overall cost increments of up to $400 \%$. As expected, these differences increase with the length of the time window.

- $\quad$ The influence of the RZ and TW parameters are larger when the number of customers is larger, despite the possibility of having more options to configure near-optimal routes, entering and leaving the restricted zone, when the number of customers increases.

- The scenario with $\mathrm{RZ}=50$ and $\mathrm{TW}=4$ shows better results than the scenario with $R Z=20$ and $T W=8$. This indicates that the influence of the length of the time window increases as the size of restricted zone grows. 
Based on the results provided by the 9 scenarios analyzed in the test problem, we can conclude that the VRPATW algorithm constitutes a sound technique to assess the introduction of access time window policies from the point of view of the extra costs imposed on carriers. We have shown the influence of the three parameters considered (number of customers to visit, size of the restricted zone and length of the time window) and the fact that a small increase in one of them does not significantly affect the results, but bigger increases in at least two of them causes relevant extra costs.

These conclusions cannot be considered obvious when these extra direct costs are neglected by local authorities when implementing access time window policies, while considering externalities like pollution, congestion or visual intrusion. The contribution of Operations Research techniques provides a powerful tool to evaluate these extra costs and incorporate them to the analysis. The illustration of the model with the test problem shows the need for more vehicles and more route time in the case of no time window scenario. The implications of this fact in terms of congestion and pollution may thus be evaluated. Future research along this line contemplates the application of this model to actual cities where a time window policy is implemented, in order to estimate the additional emissions, congestion and costs induced by it.

\section{References}

[1] Anderson, S., Allen, J. and Browne, M., Urban logistics - how can it meet policy makers sustainability objectives?, Journal of Transport Geography, 13, 71-81, 2005.

[2] Seasons, M., Monitoring and Evaluation in Municipal Planning, Journal of the American Planning Association, 69(4), 430-440, 2003.

[3] Vickerman, R., Evaluation methodologies for transport projects in the United Kingdom. Transport Policy, 7, 7-16, 2000.

[4] Uchimura, K. and Sakaguchi, H., Vehicle routing problem using genetic algorithms based on adjacency relations. Vehicle Navigation and Information Systems Conference. Proceedings in conjunction with the Pacific Rim TransTech Conference. 6th International VNIS. 'A Ride into the Future', Seattle, WA, USA, 214-217, 2002.

[5] Donati, A.V., Montemanni, R., Casagrande, N., Rizzoli, A.E. and Gambardella, L.M., Time dependent vehicle routing problem with a multi ant colony system, European Journal of Operational Research, 185, 11741191, 2008.

[6] Figliozzi, M.A., The impacts of congestion on commercial vehicle tour characteristics and costs, Transportation Research E, 46, 496-506, 2010.

[7] Fleischmann, B., Gnutzmann, S. and Sandvoß, E., Dynamic Vehicle Routing Based on Online Traffic Information, Transportation Science, 38(4), 420-433, 2004.

[8] Quak, H. and De Koster, M.B.M., Delivering Goods in Urban Areas: How to Deal with Urban Policy Restrictions and the Environment, Transportation Science, 43(2), 211-227, 2009. 